\title{
An inexpensive integrated circuit intracranial stimulator
}

\author{
ALAN J. FRIDLUND and MARK R. LEBERER \\ The University of Mississippi, University, Mississippi 38677
}

\begin{abstract}
An intracranial stimulation unit which offers precise control of stimulus duration, frequency, and current is described. The unit generates a square wave stimulus output utilizing very inexpensive, easily constructed integrated circuitry.
\end{abstract}

Commercially available stimulators which allow the experimenter to control pulse train frequency, duration, and amplitude may cost several hundred dollars or more. ${ }^{1}$ This report describes an integrated circuit stimulator that permits control of the above parameters, yet can be constructed for under $\$ 25.00$. The chief constraint of the proposed circuit is that the stimulus waveform is not selectable; the pulse train provided is a fixed $50 \%$ duty cycle biphasic square wave. More elaborate waveforms, such as the biphasic pulse-pair advocated by Lilly (1955), require more elaborate circuitry. However, the $50 \%$ duty cycle square wave employed in the present design has two advantages when compared with shorter pulses: it allows the use of lower voltages to achieve required coulomb thresholds at the electrode site (Mickle. 1961); and, it facilitates computation of peak current reaching the electrode site during stimulation. This design can be used in student laboratories and general experimental work where variation of the stimulus waveform is not necessary.

A schematic of the stimulator is shown in Figure 1. The circuit utilizes two LM 555 integrated circuits ${ }^{2}$, one in monostable (IC1) and one in astable (IC2) multivibrator configuration. Upon shunting of the trigger input to ground (by the closing of a microswitch, for example), IC1 is set to produce a pulse of given duration, determined by $R_{D}$. A $10-\mathrm{k} \Omega$ resistor prevents spurious triggering by "pulling up" the open trigger input of IC1 Pin 2, to $+\mathrm{V}$.

The R-C circuit formed by the 2.2-M $\Omega$ resistor and $.015-\mu \mathrm{F}$ capacitor limits the triggering to only one pulse from ICI regardless of the length of time that the trigger inputs are shunted. The sustain switch bypasses the R-C circuit and allows continuous-duty operation of IC1 by grounding input Pin 2 .

The pulse from IC1 $P$ in 3 powers both the stimulus on light-emitting diode (LED 2) and astable IC2. IC2

This research was supported in part by the Committee on Faculty Research of the Graduate School of the University of Mississippi. Requests for reprints should be addressed to Alan I. Fridlund, P. O. Box 7457. Department of Psychology, University of Mississippi. University, Mississippi 38677. generates the square-wave pulse train that constitutes the intracranial stimulus. This arrangement is fully capable of generating frequencies from $10 \mathrm{~Hz}$ to $10 \mathrm{kHz}$, depending on the selection of a suitable $\mathrm{C}_{\mathrm{F}}$.

The square wave output from Pin 3 of IC2 varies from ground to $+V$. Such a unidirectional square wave has been found to cause electrolytic tissue damage at the intracranial electrode site (Mickle, 1961). In the present circuit, the unidirectional dc (direct current) square wave is transformed into an ac (alternating current) waveform. This is achieved by referencing the Pin 3 output not to ground, but to $+\mathrm{V} / 2$ via a voltage divider circuit composed of two $2.2-\mathrm{k} \Omega$ resistors. The stimulus at the electrode is thus a true ac stimulus with voltage varying from $+\mathrm{V} / 2$ to $-\mathrm{V} / 2$. Additionally, the voltage divider circuit serves to current-limit the output of IC2 to under $5 \mathrm{~mA}$, rendering the circuit impervious to damage due to shorted stimulus outputs. The two voltage divider resistors should be closely matched to minimize dc offset at the electrode site.

Stimulus current is adjusted via the .5-MQ potentiometer. Further. the current delivered to the electrode may be read off the $0.100 \mathrm{dc}$ microammeter connected to the full-wave diode bridge as shown. The diode bridge enables the use of an inexpensive dc meter movement; ac microammeters are prohibitively expensive for this application. Since IC2 operates on a $50 \%$ duty cycle (symmetrical) square wave, current read from the meter equals one-half the current being delivered to the electrode site.

In intracranial stimulation research, it is important that stimulus current be relatively constant despite the impedance changes expected with chronic preparations. In this stimulator a large resistance, the .5-M $\Omega$ potentiometer, is placed in series with the electrode in order to adjust, and to regulate stimulus current. Any change in the electrode/tissue impedance, therefore, is a small proportion of the total load impedance presented to IC2. For example. assuming an electrode/tissue impedance of $15 \mathrm{k} \Omega$. and a setting of the potentiometer such that its resistance is $250 \mathrm{k} \Omega$, the total load impedance 


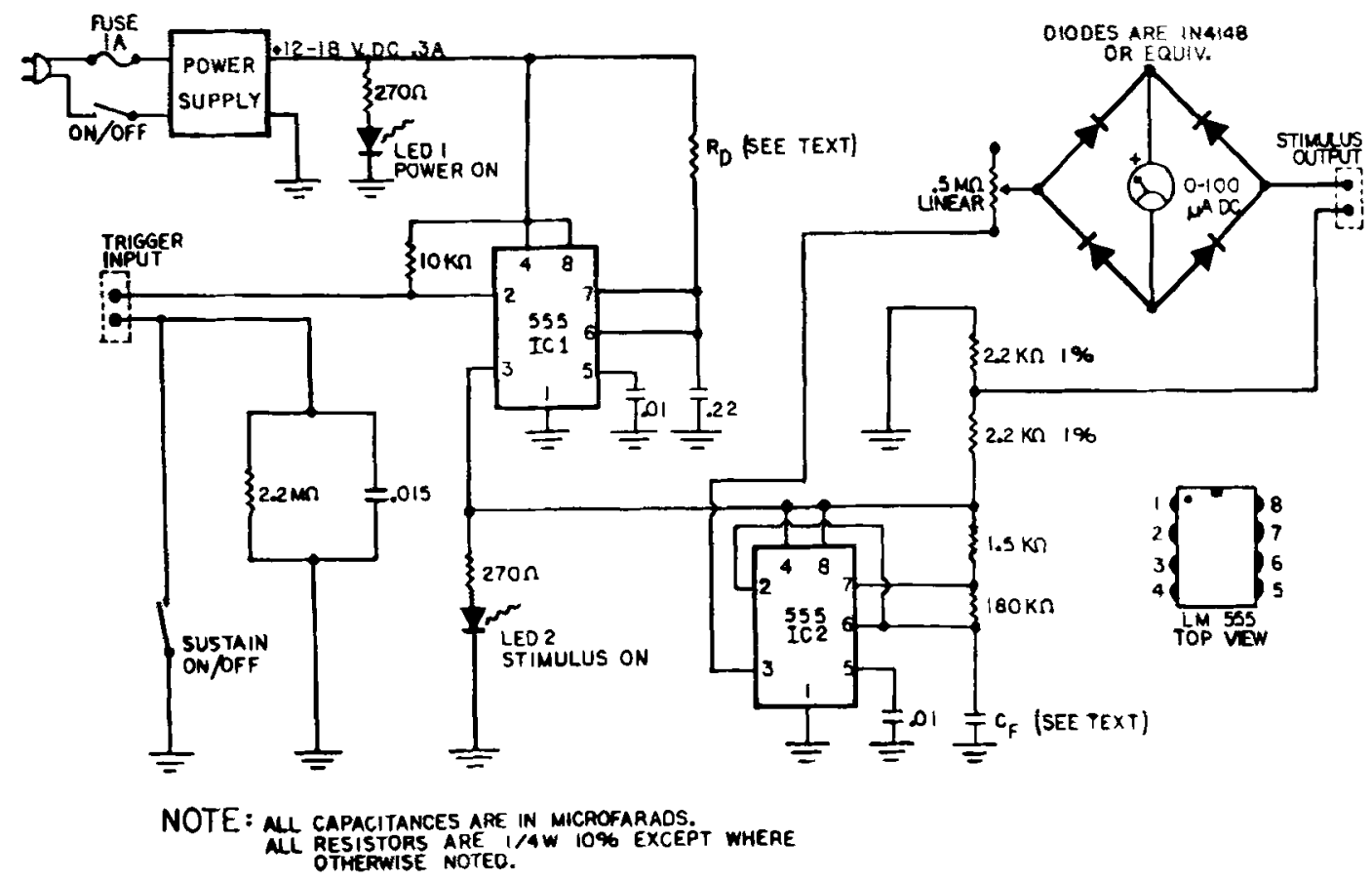

Figure 1. Circuit diagram of the intracranial stimulation unit.

presented to IC2 is $265 \mathrm{k} \Omega$. Even if the impedance of the preparation increases by one-third, to $20 \mathrm{k} \Omega$, total load impedance only increases to $270 \mathrm{k} \Omega$. Since in a series circuit current in any part of the circuit is a function of the total circuit impedance, current at the electrode site decreases by only $2 \% \quad[(256 \mathrm{k} \Omega$ $270 \mathrm{k} \Omega) / 265 \Omega$ ]. Thus for practical intensities of stimulation, current regulation is quite satisfactory. The operation of IC 2 itself is relatively independent of wide variations in electrode impedance.

Power supply requirements for this circuit are not critical. Any 12-18 V dc supply capable of producing $305 \mathrm{~mA}$, and with some filtering, will work. The circuitry is compact and, excluding power supply, can easily be placed on one $4 \times 6$ in. printed circuit card. although lead dress is not critical and point-to-point wiring may be used effectively. If possible, the stimulator ground should "float" free from the conductive elements of the animal's cage. ${ }^{3}$

Table 1

Values for $C_{F}$ for Varying Stimulus Frequencies

\begin{tabular}{cc}
\hline$C_{F}$ (Microfarads) & Frequency $(\mathrm{Hz})$ \\
\hline .0033 & 1000 \\
.005 & 750 \\
.0075 & 500 \\
.01 & 350 \\
.02 & 200 \\
.027 & 120 \\
.033 & 100 \\
.075 & 60 \\
\hline
\end{tabular}

Stimulus duration and frequency are determined by appropriate selection of duration resistor $R_{D}$ and frequency capacitor $C_{F}$. These parameters have been shown to influence substantially responses induced at specific electrode sites (Ervin \& Kenney, 1971).

In our laboratory. the prototype stimulator employed six-position switches for $R_{D}$ and $C F$. such that stimulus duration could be varied from $.05-1.0 \mathrm{sec}$, and frequency from $60 \mathrm{~Hz}$ to $1 \mathrm{kHz}$.

$C_{F}$ should be determined first. Use a temporary value of $1 M \Omega$ for $R_{D}$. Close the sustain switch and shunt the trigger inputs; this turns IC2 on indefinitely. The use of a digital frequency counter is highly recommended, and is connected to the stimulus outputs for this measurement. If such a counting meter is not available. the stimulus outputs may be connected directly to $600-\Omega$ head phones. The stimulus frequencies can then be calibrated with an audio generator by "beating" the stimulus output frequency against the generator frequency. The frequencies match when the beat frequency is $0 \mathrm{~Hz}$. Values of $C_{F}$ are selected for the frequencies desired; approximate values for different frequencies are shown in Table 1 . Precise frequency determinations will require addition of appropriate "gimmick" capacitors either in series or parallel with CF. In general. a close approximation for $\mathrm{CF}_{\mathrm{F}}$ an be obtained from the formula:

$$
F(H z)=\frac{1}{251 K\left(C_{F}\right)}
$$


Table 2

Values of $R_{D}$ for Varying Stimulus Durations

\begin{tabular}{cc}
\hline$R_{D}($ Ohms) & Duration (Sec) \\
\hline $220 \mathrm{~K}$ & .05 \\
$430 \mathrm{~K}$ & .1 \\
$850 \mathrm{~K}$ & .2 \\
$1.3 \mathrm{meg}$ & .3 \\
$1.7 \mathrm{meg}$ & .4 \\
$2.0 \mathrm{meg}$ & .5 \\
\hline
\end{tabular}

Inexpensive disk capacitors were employed, and yielded a frequency stability of $\pm 3 \mathrm{~Hz}$. Stability may be greatly improved by using more expensive mylar capacitors.

Precise determination of $R_{D}$ is best accomplished using a digital counter. The sustain switch is opened. The counter is again connected to the stimulus outputs. The number of square wave pulses for a given frequency (already established by $C_{F}$ ) and duration is calculated. For instance, if the operating frequency of IC 2 has been chosen to be $100 \mathrm{~Hz}$, then a .5 -sec stimulus duration will yield 50 square wave pulses at the outputs, for each triggering event. Thus the correct value of $R_{D}$ is that which will produce a count of 50 on the digital counter each time the trigger inputs are shunted. Approximate values of $R_{D}$ for varying stimulus durations are noted in Table 2 . In general the appropriate value for $R_{D}$ can be calculated using the formula:

$$
R_{D}=\frac{D_{S}}{.235},
$$

where $R_{D}$ is expressed in megohms and $\mathrm{DS}$ is the desired stimulus duration in seconds.

Table 3 includes all the componentry needed to construct the stimulation unit.

It should be noted that a pulse indicating stimulus onset and duration may be derived from Pin 3 of IC1. This pin is normally at ground potential, and rises to $+\mathrm{V}$ during stimulation. The pulse may be used directly, or divided as appropriate, for input to an event recorder, computer interface, or any other device which draws no more than $50 \mathrm{~mA}$.

The trigger inputs may also be connected directly to a TTL interface; TTL " 0 " activates the stimulator. Thus, stimulation can easily be brought under computer control.
Table 3

Parts List for the Intracranial Stimulation Unit

\begin{tabular}{cl}
\hline Quantity & \multicolumn{1}{c}{ Component } \\
\hline 2 & LM555 integrated circuit \\
4 & 1N4148 silicon diode (or equivalent) \\
2 & light emitting diode \\
2 & $270 \Omega 1 / 4 \mathrm{~W} 10 \%$ resistor \\
1 & $1.5 \mathrm{k} \Omega 1 / 4 \mathrm{~W} 10 \%$ resistor \\
2 & $2.2 \mathrm{k} \Omega 1 / 4 \mathrm{~W} 1 \%$ resistor \\
1 & $10 \mathrm{k} \Omega 1 / 4 \mathrm{~W} 10 \%$ resistor \\
1 & $180 \mathrm{k} \Omega 1 / 4 \mathrm{~W} 10 \%$ resistor \\
1 & $2.2 \mathrm{M} \Omega 1 / 4 \mathrm{~W} 10 \%$ resistor \\
1 & $.5 \mathrm{M} \Omega$ linear taper potentiometer \\
1 & R (see text) \\
2 & $.01 \mu \mathrm{F} 50 \mathrm{~V} \mathrm{dc} \mathrm{(ceramic} \mathrm{or} \mathrm{mylar)}$ \\
1 & $.015 \mu \mathrm{F} 50 \mathrm{~V}$ dc (ceramic or mylar) \\
1 & $.22 \mu \mathrm{F} 50 \mathrm{~V}$ dc (ceramic or mylar) \\
1 & CF (see text) \\
1 & Power supply, 12-18 V dc, 300 mA \\
2 & SPST toggle switches \\
1 & $0-100$ dc microammeter \\
\hline
\end{tabular}

\section{REFERENCES}

Ervin, F. R., \& Kenney, G. J. Electrical stimulation of the brain. In R. D. Myers (Ed.). Methods in psychobiology. New York: Academic Press, 1971. Pp. 233-237.

Lilly. J. C.. Hughes, J. R., Alvord, E. C.. JR., \& Galkin, T. W. Brief. noninjurious electric waveform for stimulation of the brain. Science, 1955, 121, 468-469.

Mickle, W. A. The problem of stimulation parameters. In D. E. Sheer (Ed.), Electrical stimulation of the brain. Austin. Texas: The University of Texas Press, 1961. Pp. 64-67. 70 .

\section{NOTES}

1. Exemplary are the Haer $4 B$ Digital Stimulator with Optical Isolator 74-60-1 (5945.00); and the W-P Instruments Model 302-T Anapulse Stimulator, with two Model 305-2 stimulus isolation units to obtain a bipolar waveform $(\$ 1,410.00)$.

2. The LM 555 integrated circuit is commonly available at Radio Shack outlets at $\$ 1.49$ per chip. The IC is also available as NE $555 \mathrm{~V}$ from James Electronics, P. O. Box 882, Belmont, California 94002 , at $\$ .70$ per chip. The $555 \mathrm{IC}$ is also available as a dual chip. LM $556 \mathrm{~N}$. It is not recommended for this application, however. since it is more expensive than two LM 555s and since failure of one section of the 556 necessitates replacement of the entire IC.

3. "Floating" the stimulator ground from the cage floor and manipulanda assures that no $D C$ offet voltage will arise during stimulus presentation, due to the offset correction induced by the voltage divider circuitry. Studies in our laboratory, however, indicate that any offset introduced from the electrode site through the animal's body to a grounded cage floor is negligible, amounting to offsets less than 3 microA.

(Revision received for publication December 30, 1975; accepted January 3, 1976.) 